\title{
基于叶片识别的侵入式室内植物建模
}

\author{
史川石，郑倩*，黄惠 \\ (深圳大学可视计算研究中心 深圳 518060) \\ (qianzheng85@gmail.com)
}

\begin{abstract}
摘 要: 由于室内植物的叶片存在大量自遮挡, 为了得到植物的完整三维信息, 往往需要用户手动裁剪、扫描和配准 叶片. 针对该问题，提出了利用实例分割网络进行叶片识别并选取被裁剪叶片的方法. 通过总结植物叶片形状与分 布特征，建立虚拟植物模型，渲染大量带叶片轮廓信息的图片来训练实例分割网络，避免了耗费大量精力标注真实 植物叶片. 进一步提出了自动化建模系统, 根据单个视角下观察到的植物图像自动选择被裁剪的叶片, 并获得该叶 片的三维模型; 调整相机位置让更多的叶片被检测到并剪掉，以恢复植物的三维信息. 使用该系统对绿萝叶片、鸭脚 木和花烛 3 种虚拟植物进行了测试, 并评估重建的整株植物重合度、叶片重合度和叶片占比, 结果显示使用本文方法 重建的植物模型能更完整地恢复原始植物的三维信息.
\end{abstract}

关键词: 植物建模; 侵入式建模; 叶片检测; 实例分割

中图法分类号: TP391.41 DOI: 10.3724/SP.J.1089.2021.18342

\section{Intrusive Indoor Plant Modeling Guided by Leaf Recognition}

\author{
Shi Chuanshi, Zheng Qian", and Huang Hui \\ (Visual Computing Research Center, Shenzhen University, Shenzhen 518060)
}

\begin{abstract}
Due to the heavy self-occlusion of indoor plants, to capture the complete 3D information of a plant, users usually need to manually cut the leaves, scan them, and register the leaf scans together. In response to this problem, we propose a method to identify and select the leaves to crop using the instance detection network. To avoid manually labeling photos of real plant leaves for training, we summarize the leaf shape and distribution to build 3D plant models, then render lots of images with leaf contour information. An automatic intrusive reconstruction system for capturing the full $3 \mathrm{D}$ of indoor plants is introduced, which automatically and successively selects a leaf to cut from a captured image to obtain its 3D points and adjusts the camera view when nothing is detected. Three types of virtual indoor plants have been tested and evaluated. Results show that the reconstructed plant models capture the plants well.
\end{abstract}

Key words: plant modeling; intrusive modeling; leaf detection; instance segmentation

三维植物建模是计算机图形学里一个具有广 泛应用的重要议题. 植物模型在真实感游戏场景 设计、物理模拟与渲染中起重要作用; 在植物学与
农业领域, 植物模型与植物生长模拟、农作物施肥 与害虫管理等方面有紧密的联系. 目前常见的三 维植物建模方法是通过扫描设备扫描植物得到颜

收稿日期：2020-05-03; 修回日期：2020-09-08. 基金项目：国家自然科学基金(61861130365, U2001206); 广东省自然科学基 金 (2020A0505100064，2015A030312015); 广东省高等学校科技创新重点项目 (2018KZDXM058); 深圳市基础研究基金 (JCYJ20180305125709986). 史川石(1992一), 男, 硕士研究生, 主要研究方向为计算机图形学; 郑倩(1985一), 女, 博士, 助理教授, CCF 会员, 论文通讯作者, 主要研究方向为计算机图形学; 黄惠(1977一), 女, 博士, 教授, 博士生导师, CCF 杰出会员, 主要研究方 向为计算机图形学. 
色和深度信息, 再通过三维重建得到植物模型. 但 由于植物的外部枝叶会不同程度地遮挡内部枝叶, 因此很难得到植物的完整信息. 完整准确地得到 植物模型是一个有挑战的工作.

对于室外植物, 人们更多地关注于它们的枝 干结构, 这可以通过三维扫描得到. 而对于室内植 物而言, 人们更关注它们的叶片形状与分布, 但内 部叶片会被外部叶片遮挡，导致无法获得准确的 植物模型. 因此室内植物的自遮挡性质会更加影 响人们对它们的认知. 为了得到被遮挡部分的叶 片信息，人们需要剪掉外部叶片让内部叶片可见. 由于这一过程破坏了现实中的植物，因此称为侵人 式建模方法 ${ }^{[1]}$. 虽然侵人式建模方法毁坏了现实中 的植物，但让植物的内部叶片信息可见，使完整准 确的植物建模成为可能. 但是传统的侵人式建模方 法通过经验手动选取被剪切的叶片，剪切叶片并扫 描, 再配准扫描数据, 操作过程烦琐且扩展性差.

近年来, 随着深度学习方法的逐渐流行, 研究 者也使用深度学习方法检测植物种类、区别作物与 杂草、检测虫害叶片. 本文利用深度学习方法, 通 过实例分割网络从植物图片中检测叶片并剪掉, 获取叶片点云, 再将叶片模型与点云配准. 在剪掉 所有叶片后，通过组合叶片模型得到完整的室内 植物模型. 为了验证该方法, 对自遮挡现象普遍的 3 类室内植物模拟侵人式建模; 给出了 3 种评估准 则对建模结果进行评估, 并讨论本方法在多种植 物建模时的适用性.

\section{1 相关工作}

\section{1 植物建模}

过去几十年里, 植物三维建模方法一直是计 算机图形学中的热点研究方向, 研究者通过定义 语法、草图勾勒、二维图像、点云重建等手段进行 植物建模. Honda ${ }^{[2]}$ 在 1971 年提出将树木的枝干定 义为递归的树状结构. 1994 年, Prusinkiewicz 等 ${ }^{[3]}$ 提 出文本描述规则的 L 系统 (L-System), 通过定义规 则文本递归地从初始状态逐渐生成一株完整植物. 1999 年, Lintermann 等 ${ }^{[4]}$ 提出 xfrog 系统, 为了便于 用户使用，定义符合树木外形的规则用于建模. 2001 年, Shlyakhter 等 ${ }^{[5]}$ 采集了一株植物的多幅图 片, 利用可视外壳思想计算树木的三维外形轮廓 并提取主要枝干信息，其余部分由 $\mathrm{L}$ 系统生成. 2005 年, Ijiri 等 ${ }^{[6]}$ 在基于草图的基础上，通过交互 式方法并基于 L 系统进行植物建模. 2008 年, Chen
等 ${ }^{[7]}$ 从二维枝干草图触发, 在模型库中搜索最匹配 草图的样本，然后使用概率模型构建出三维信息. 2010 年, Livny 等 ${ }^{[8]}$ 针对扫描得到的植物信息, 使 用全局优化方法重建被遮挡的植物枝干.

针对植物的自遮挡性质，研究者一般利用已 知的先验知识来指导建模. 对于室外植物, 可以利 用启发式方法提取树木的枝干结构，然后进一步 随机生成末梢细节的几何网格模型 ${ }^{[9]}$; 或者从树 叶与枝干不同的散射属性出发, 根据点云的密度 信息来提取主枝干和树叶 ${ }^{[10]}$. 对于室外植物, 2013 年, $\mathrm{Li}$ 等 ${ }^{[11]}$ 利用激光点云连续扫描植物的生长, 以 准确地定位室内植物发芽和分枝的时刻和位置, 但是此方法需要大量先验知识, 无法快速地应用 于一株给定的室内植物中. 研究者也考虑能够扫 描到室内植物的内部叶片, 这样可以获得更准确 的叶片信息. 在使用三维扫描仪扫描植物时, Yan 等 ${ }^{[12]}$ 提出人为辅助移开外部遮挡的叶片, 从而获 取内部的植物枝叶信息，但其并不能处理具有复 杂遮挡关系的植物. 随后, Yin 等 ${ }^{[1]}$ 提出通过一定 的顺序剪掉植物的叶片, 让处于内部的枝叶逐渐 可见, 最终使整个植物的几何结构暴露出来, 实现 消除自遮挡的目的. 2016 年, Xie 等 ${ }^{[13]}$ 提出将真实 树木模型分割成若干模块, 这些模块可用于引导 构建植物模型, 并且同时借助植物学中的知识来 控制树枝的相对大小. 2017 年, Beardsley 等 ${ }^{[14]}$ 通过 对一张有很多叶片的图片进行超像素分割得到每 个单独叶片后, 通过参数化叶片模型匹配到三维 点云上，实现了快速重建一个具有大量叶片的场 景的方法. 但是这些方法均需要手动剪掉叶片, 工 作烦琐且难以扩展，因此自动选取剪掉的叶片是 相关研究的方向之一

\section{2 实例分割网络}

在计算机视觉领域, 将目标与杂物分开是自 动目标识别中最基本的问题. 1998 年, LeCun 等 ${ }^{[15]}$ 提出由 3 个全连接层组成的 LeNet, 并在字母识别 中取得了很好的结果. Girshick 等分别在 2014 年和 2015 年提出了区域卷积神经网络 $(\mathrm{R}-\mathrm{CNN})^{[16]}$ 和 Fast R-CNN ${ }^{[17]}$, 其核心思想是使用非深度的方法 在图像中提取图形块, 然后使用深度学习将这些 图像块放到一个具体物体的位置. 2017 年, $\mathrm{He}$ 等 ${ }^{[18]}$ 提出了 Mask R-CNN，在特征提取方面采用已有的 架构, 额外添加了 Mask 预测分支, 使神经网络在 实例分割方面有了长足应用. 同年, $\mathrm{Cai}$ 等 ${ }^{[19]}$ 提出 了 Cascade R-CNN，对预测框与检测框的交集采用 不同的阈值, 从而训练了多个级联的检测器, 解决 
了在检测过程中检测框容易被噪声干扰的问题. 2019 年, Chen 等 ${ }^{[20]}$ 提出了混合任务的 Cascade 网 络, 将 Mask R-CNN 与 Cascade R-CNN 结合起来, 交替进行包围盒预测与掩码预测，在不同大小的 模型上检测性能都有非常稳定的提升. 随着深度 学习技术的发展, 神经网络与其他学科结合的应 用逐渐发展，尤其是在植物学、农业相关领域有很 广的应用. 2017 年, Amara 等 ${ }^{[21]}$ 提出了使用 LeNet 作为检测叶片的手段, 用于区分健康与遭到病害 的香蕉树叶子. 2018 年, Joffe 等 ${ }^{[22]}$ 提出了一个叶片 采样系统, 只使用单目相机和 6 自由度机械臂就可 以采摘健康和不健康的叶片, 而不需要利用植物 的先验知识.

虽然深度学习方法已有大型通用数据集，但 是在具体场景下往往需要特定的数据集, 当数据 获取难度较大或工作量过大时, 往往需要自定义 生成数据集. 2017 年, Georgakis 等 ${ }^{[23]}$ 针对室内场 景，通过分析场景中可用于支撑的平台，在场景中 适当位置添加可摆放物体，生成了虚拟的室内场 景图片. 而在植物相关的研究中, 2018 年, Ward 等 ${ }^{[24]}$ 通过总结拟南芥的叶片分布规律, 将叶片三维模 型放置在合适的位置并渲染得到虚拟的拟南芥图 片. 2019 年, Kuznichov 等 ${ }^{[25]}$ 从莲座叶丛植物图片 中提取叶片, 随后旋转、平移并重新组合这些叶片, 并添加与原始图片类似的背景, 合成虚拟植物叶 片数据集, 用于训练神经网络.

\section{2 叶片识别}

侵人式植物建模的关键点在于建模时定位合 适的叶片并剪掉. 可以通过使用实例分割网络从 任一视角的植物照片中识别处于植物外部的叶片. 训练实例分割网络时需要大量标注了叶片轮廓的 图片, 但直接对真实植物拍摄大量照片并标注要 消耗大量的时间与人力, 所以本文中使用虚拟植 物模型渲染得到的图片来训练网络. 虚拟植物中 的叶片模型全部已知, 通过计算得到每个叶片的 轮廓对应到图片中的像素位置, 即可直接合成叶 片轮廓标注信息, 比手动标注降低了工作量.

\section{1 生成训练数据}

目前已有一些公开的植物叶片数据集, 例如, 美国的加利福尼亚大学欧文分校发布的 UCI Leaf Dataset 数据集 ${ }^{[26]}$ (如图 1a 所示)中包含 40 种不同植 物叶片; 美国的哥伦比亚大学和马里兰大学合作

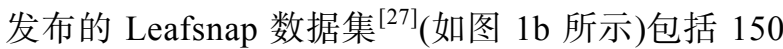

多种美国东北部的植物的叶片; 瑞典林雪平大学 发布的瑞典叶片数据集 ${ }^{[28]}$ 包含 15 种瑞典常见的树 木的叶片. 但是这些数据集都只有单个叶片图像, 适用于分类问题; 而且这些数据集的叶片与本文 所需要的室内植物叶片差异过大, 并且没有不同 叶片之间的关系. 因此这些数据集不能直接应用 于本项目, 本文需要生成叶片数据集. 手动标注图 片中的叶片轮廓耗时、耗力, 并且由于光照等原因 使得图片明暗度不同，导致很难准确地标注叶片. 因此通过参数化叶片模型模拟叶片形状, 并根据 真实植物中叶片的分布情况生成虚拟植物，随后 生成虚拟叶片数据用于训练神经网络.

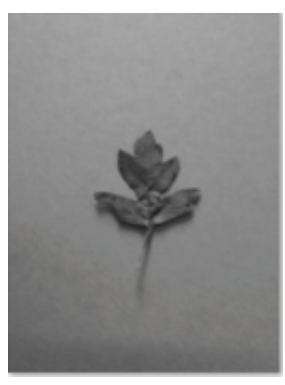

a. UCI Leaf Dataset

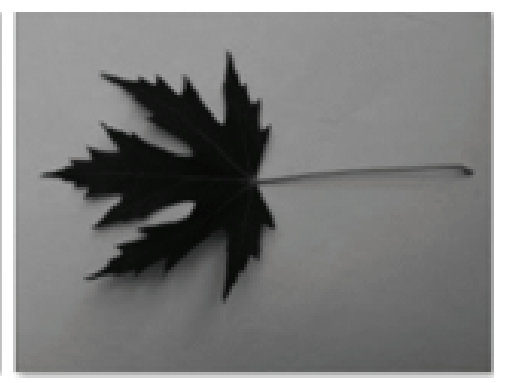

b. Leafsnap 数据集
图 1 现有的植物叶片数据集

为了让模拟的虚拟植物与真实植物尽可能地 接近, 需要在模拟时考虑虚拟植物与真实植物在 2 个方面相似性, 即叶片模型外形相似与叶片分布 相似. 为了使模拟所用的叶片模型与真实植物叶 片相似, 本文采用 Bézier 曲线定义的参数化叶片 模型 ${ }^{[14]}$, 通过调整参数使叶片模型与真实植物相似.

本文分别对绿萝、鸭脚木和花烛 3 种室内植物 进行模拟. 绿萝植物外形如图 2a 所示, 其叶片形 状为桃心形. 绿萝的主干可以看做一个圆柱, 叶片 基本围绕中心主干分布; 叶片按层分布, 每层内的 叶片则绕主干旋转分布; 除去植物顶部与底部这 些特殊位置, 叶片往往与地面呈一定的角度倾斜. 基于此观察, 叶片可简化为在坚直方向上层级分 布, 每一层叶片围绕植物中心主干旋转分布. 模拟 得到的虚拟绿萝如图 $2 b$ 所示.

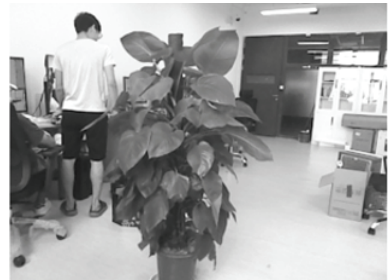

a. 真实绿萝

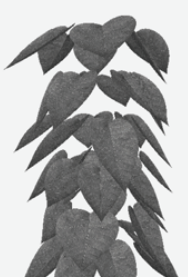

b. 虚拟绿萝
图 2 真实绿萝与虚拟绿萝 
鸭脚木也是常见的室内植物, 它的叶片形状 与整体外形如图 3a 所示. 其叶片近似于椭圆, 叶 片底部更加尖锐, 最主要特征是一组叶片 (7 9 片) 会绕一个中心点旋转分布. 一株鸭脚木有多个这 样的叶片组，它们的分布符合植物枝干之间对空 间、光照等资源的竞争关系，既不会过分稀疏也不 会过分密集. 模拟得到的虚拟鸭脚木如图 $3 b$ 所示.

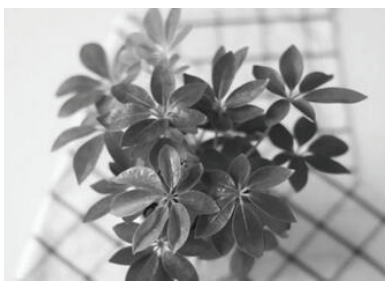

a. 真实鸭脚木

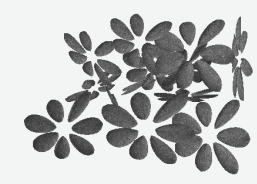

b. 虚拟鸭脚木

图 3 真实鸭脚木与虚拟鸭脚木

花烛的叶片形状与绿萝叶片相似; 一株花烛 有 8 12 片叶子，叶片符合常见的植物叶片对空间 的竞争关系, 花烛会有红色的花, 但本文中只考虑 叶片建模，因此模拟时会忽略花. 模拟得到的虚拟 花烛如图 4b 所示.

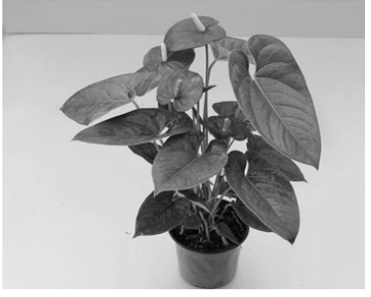

a. 真实花烛

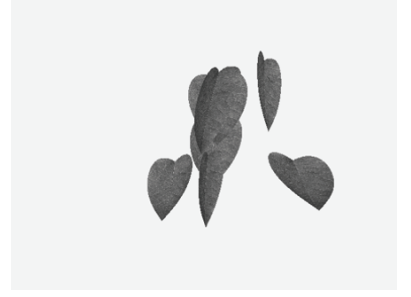

b. 虚拟花烛
图 4 真实花烛与虚拟花烛

在构建植物模型后，利用计算机图形学中投 影的知识, 可以很方便地根据三维模型计算得到 它们在渲染出的图片中所占据的像素位置. 因此 本文方法关键在于获得虚拟植物中合适的叶片. 为了更加接近现实中根据观察到的情况选取叶片 的行为, 本文中只标注出处于植物外部、能被完整 观察到且正面朝向观察点的叶片.

通过计算叶片朝向与虚拟相机投影方向的夹 角能判断叶片是否正面朝向观测点. 一个叶片模 型的朝向 $\boldsymbol{n}=\sum_{v=1}^{V} w_{v} \boldsymbol{n}_{v}$ 可近似为其所有顶点的法向 量加权. 其中, $V$ 表示叶片模型中顶点数量; $v$ 表 示模型中的每一个顶点; $\boldsymbol{n}_{v}$ 为每一个顶点的法向 量； $w_{v}$ 为对应的归一化后的权重，由其 1 阶邻域的 面积之和决定. 当叶片朝向 $\boldsymbol{n}$ 和相机观察方向夹角 小于给定的阈值 $\alpha$ 时, 即认为叶片正面朝向相机.
在确定叶片是否被遮挡时, 利用深度缓存中 的值判断叶片之间的遮挡关系. 考虑一株植物中 完整地显示整个叶片的情况过于稀少，本文定义 只要叶片的可见区域大于某一阈值 $\beta$, 则认为该 叶片可见.

\section{2 选择实例分割网络}

图像上的物体分割是一个常见的问题，在计 算机视觉领域，对象分类、语义分割、对象检测和 实例分割均是相关的课题. 本文将某一视角下可 以看到的植物信息存储为一张 RGB 图片, 通过这 张图片得到要剪掉的叶片; 使用 MMDetection ${ }^{[29]}$ 作为检测框架，选用 Mask R-CNN 作为实例检测网 络, 在某一视角下检测出多片叶子, 选择置信度最 高的一片进行裁剪.

\section{3 叶片处理}

对于虚拟植物，在使用实例分割网络检测图 片中的叶片时, 检测到可见叶片后得到的是一张 二进制图片. 直接通过检测结果像素对比来剔除 虚拟植物中叶片的效率较低, 如果能快速地根据 检测到的叶片所属像素找到所对应叶片索引, 将 会加速这一过程.

本文引人叶片索引图，即将叶片索引线性地 映射到 RGB 空间中, 生成一张不同叶片由不同颜 色标注出的图片. 将叶片索引映射为 RGB 色彩的 公式为

$$
C_{i}= \begin{cases}255, & \text { if } \varphi>N \times i \\ (\varphi \bmod N) \times \sigma, & \text { otherwise }\end{cases}
$$

其中, $C_{i}, i \in\{1,2,3\}$ 表示对应的 RGB 颜色通道的 值; $\varphi$ 为生成的虚拟植物中叶片在数组中的索引; $N$ 为每个颜色通道可以表示的叶片个数, 由 $N=256 / \sigma$ 计算得到, 其中 $\sigma$ 为定义的叶片索引 之间的颜色间隔值. 虽然索引颜色只要有微弱差 异就能被系统分辨，但通过设置合适的颜色间隔 $\sigma$, 让不同叶片之间颜色差异更明显，这样更便 于结果观察. 图 5 展示了一株虚拟绿萝在某一视角 下渲染的植物图片与叶片索引图片, 可以看出叶 片索引与原始叶片有良好的对应关系。

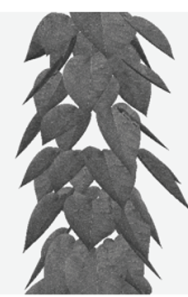

a. 分块结果虚拟植物图片

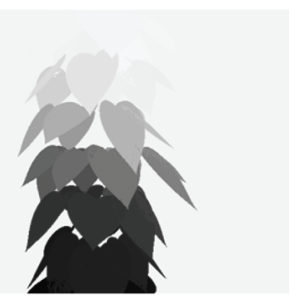

b. 叶片索引图片
图 5 虚拟植物图片与叶片索引图片 
实例分割网络在接收到叶片索引图片之后, 需要对色彩进行解码. 色彩解码的过程为编码的 逆过程, 具体公式为

$$
\varphi=\sum_{i=1}^{3}\left\lfloor C_{i} / \sigma\right\rfloor .
$$

通过将 $C_{i} / \sigma$ 的值进行向下取整, 使得到的索引值 为整数, 保证了 $\mathrm{RGB}$ 颜色与索引的正确对应. 将 对虚拟植物图片检测到的二进制图片与叶片索引 图片进行比对, 即能得到虚拟植物中需要剔除的 叶片的索引.

\section{3 虚拟植物建模}

在植物图片中检测到图片并可以将其剪掉后, 就可以对一株植物进行侵人式建模. 本文提出了 客户端-服务器形式的自动化建模系统, 将建模过 程分解为三维模型操作与二维图片操作. 此外还 定义 3 种评估建模结果的方式, 用于分析建模结果.

\section{1 系统流程}

对虚拟植物建模的整体流程如图 6 所示, 对一 株虚拟植物, 首先在初始视角下渲染得到该植物 的图片, 并通过网络检测叶片, 当检测到叶片时, 去除该叶片并获得叶片点云, 进行叶片配准; 之后 继续在此视角下检测; 否则调整相机位置重新渲 染. 重复上述过程直至所有叶片都被剔除, 组合所 有叶片模型, 最终得到完整的植物模型.

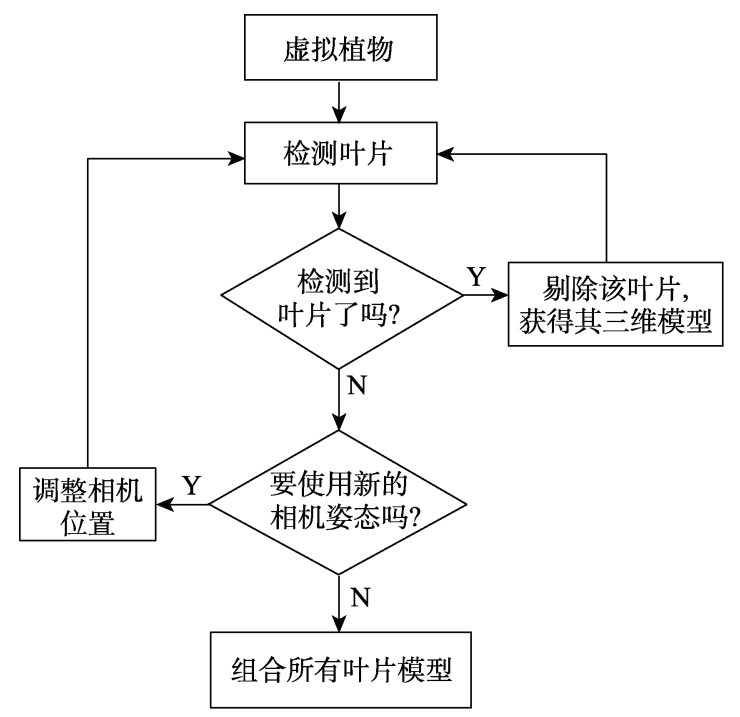

图 6 虚拟植物建模流程图

在整个流程中使用实例分割网络检测叶片只 对 RGB 图片进行操作, 而与虚拟植物相关操作并 无相关性. 因此, 提出将图片的操作与虚拟植物操
作分离. 系统的服务器部分主要包括接收图片、处 理图片信息、实例分割网络进行检测、返回检测结 果等功能和操作; 剩余是对虚拟植物的操作过程, 包括构造虚拟植物图片、生成虚拟图片、剔除叶片、 调整相机位置等整合为客户端部分.

首先在服务器端加载训练好的实例分割网络 的检测模型, 再使用 socket 创建服务器并绑定 IP 地址与端口, 然后检测接收的被检测图片. 服务器 端接收 RGB 图片与叶片索引图片, 通过实例分割 网络对 RGB 图片进行可见叶片检测, 如果检测到 了可见叶片, 将检测结果(一张二进制图片)与叶片 索引图片进行对比, 得到需要剔除的叶片的索引, 并将该索引返回给客户端. 如果在 RGB 图片中没 有检测到可见叶片, 那么服务器端直接返回一个 标识符给客户端. 之后服务器继续等待下一次传 输任务; 收到图片后再执行检测叶片、返回叶片索 引等操作过程.

在客户端部分，首先按照设定好的规则生成 虚拟植物模型, 然后在某一初始位置使用虚拟相 机获取当前植物的 RGB 图片、叶片索引图片以及 深度图片, 其中 RGB 图片与叶片索引图片被发送 至服务器, 之后等待服务器返回叶片索引. 如果接 收的服务器返回数据为没有检测到叶片的标识符, 则在客户端中调整相机参数, 重新生成 3 张图片并 发送其中的 RGB 图片与索引图片. 如果接收的是 正确的叶片索引, 则将虚拟植物中对应的叶片剔 除, 仍然在当前视角下生成图片并进行后续操作.

在植物建模时, 某一视角下对植物模型模拟 扫描得到植物点云, 如图 7a 所示. 当剔除掉检测 到的叶片后, 可以获得植物内部信息. 图 7a 和图 $7 \mathrm{~b}$ 的红框标出了被裁剪掉的叶片区域. 借助八叉 树或 $k \mathrm{D}$ 树比较剔除前后的植物点云, 可以得到图 $7 \mathrm{c}$ 所示被剔除叶片的点云; 之后使用最近点迭代 算法将模板模型与植物点云进行配准, 得到被剔 除叶片的信息.

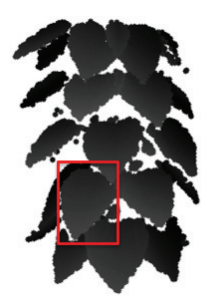

a. 剔除叶片前

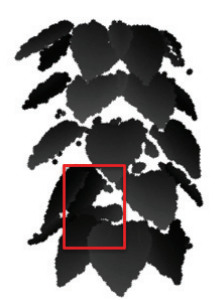

b. 剔除叶片后

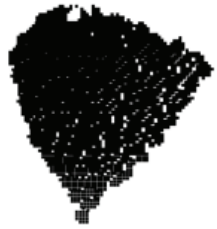

c. 被裁剪的叶片
图 7 对比剔除前后的植物点云获得叶片点云 
对于某一类植物，均可以使用同一个检测网 络模型进行检测, 图 6 中服务器的整体流程对于一 类特定的植物都是通用的. 当检测其他种类的植 物时, 加载新的检测模型即可. 通过将虚拟植物与 网络检测分开以及只对三维植物在客户端进行操 作, 服务器与客户端之间具有低耦合的特点.

\section{2 评估方式}

对虚拟植物进行侵人式建模时, 需要评估建 模结果, 即衡量得到的模型 $D$ 与原始模型 $S$ 的相 似程度. 本文以 2 个模型的重合部分占比为评估指 标, 对 2 个模型上的顶点进行评估. 如图 8a 所示, 设置一定的误差量 $r$, 对于 $D$ 上的某一点 $p_{D}$, 如 果在以该点为球心、以 $r$ 为半径的球形空间内存在 $S$ 上的点 $p_{S}$ (浅灰色的点), 则认为 $p_{D}$ 与 $p_{S}$ 重合; 否则认为不重合, 如图 $8 \mathrm{~b}$ 所示. 而在整个模型, 即 整株植物中, 则可以使用存在重合点的个数与 $D$ 中顶点个数的比例 $m_{P}$ 作为评估建模好坏的标准, 称为整株植物重合度. 进行评估的关键是选择合 适的误差量 $r$, 通过观察对比真实植物的叶片, 本 文设定 $r$ 为叶片中脉长度的 $5 \%$ 。

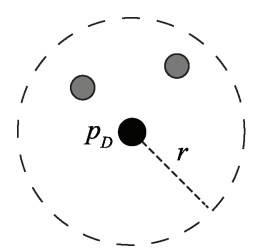

a. 黑色点与其他点重合

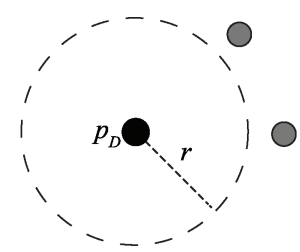

b. 黑色点与其他点不重合
图 8 评估重叠比例

在一株植物上有可能存在这样一种情况：某 片叶子的部分并没有与它所建模的原始植物中的 叶片相重合, 而是与另外一片叶子部分重合, 这样 会导致评估结果虚高. 因此本文将每一组对应的 叶片使用上面的方法进行计算，得到它们的评估 结果; 然后对所有叶片的评估结果求平均值, 称为 平均叶片重合度 $m_{L}$, 并将其作为另一评估指标.

在整个植物与每片叶子的标准上分别采用这 2 种评估指标对建模结果进行了评估, 显然平均叶 片重合度比整株植物重合度更加严格. 因此对于 叶片细节要求不高的情况, 建议采用整株植物重 合度进行评估; 而对于叶片细节要求较高的情况， 使用平均叶片重合度进行评估.

此外, 如果误差影响导致部分叶片与原始叶 片偏差较多, 则认为叶片建模结果过差, 而偏差不 多的叶片则认为建模结果较好. 建模的一株植物 中建模结果较好的叶片数量与占据总叶片数的比 例 $P_{L}$, 也作为另一评判叶片建模好坏的指标. 建
模叶片与原始叶片偏差程度依然使用上面提到的 球状空间进行判断，仅当建模叶片与原始叶片中 不少于 $70 \%$ 的点满足所定义的重合的标准，才判 断此叶片建模结果较好.

\section{4 结果与分析}

\section{1 叶片检测}

本文中训练参数使用了 MMDetection 的默认 参数: GPU 每次处理图片数为 2 , 学习率为 0.02 , epoch 为 12 , 并使用 2 张 12 GB 的 TITAN Xp 显卡 用于训练. 对于虚拟绿萝, 使用 1480 张带标注图 片作为训练集, 337 张作为验证集; 而虚拟鸭脚木 与花烛均使用 2000 张带标注的图片作为训练集, 500 张作为验证集. 虚拟植物训练时的参数与模型 准确率如表 1 所示, 其中, Bbox 和 Segm 分别代表 包围盒检测和分割检测准确率.

表 1 虚拟植物训练模型的准确率

\begin{tabular}{lccccc}
\hline \multirow{2}{*}{ 植物种类 } & \multicolumn{2}{c}{ Bbox } & & \multicolumn{2}{c}{ Segm } \\
\cline { 2 - 3 } \cline { 5 - 6 } & $\mathrm{AR}$ & $\mathrm{AP}$ & & $\mathrm{AR}$ & $\mathrm{AP}$ \\
\hline 虚拟绿萝 & 0.799 & 0.643 & & 0.825 & 0.643 \\
虚拟鸭脚木 & 0.327 & 0.327 & & 0.338 & 0.327 \\
虚拟花烛 & 0.782 & 0.779 & & 0.806 & 0.802 \\
\hline
\end{tabular}

渲染虚拟绿萝、鸭脚木与花烛得到图片的检测 结果如图 9 所示, 其中被检测到的叶片使用红色标 注, 可以看到, 在植物图片中检测到数片完整叶 片，可用于虚拟植物建模.

这 3 种植物的叶片形状和叶片分布差异很大, 因此分别训练叶片检测模型. 训练好的检测模型 可适用于同类型的植物; 对于其他类型的植物, 如 果叶片形状和分布与现有的差异比较大, 则需要 重新训练合适的检测模型.

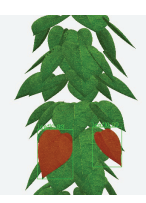

a. 虚拟绿萝

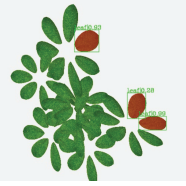

b. 虚拟鸭脚木

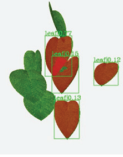

c. 虚拟花烛
图 9 绿萝、鸭脚木与花烛图片中检测到的叶片

\section{2 植物建模}

用已定义的客户端-服务器建模系统, 可以模 拟虚拟植物的建模过程. 表 2 展示了进行建模的虚 拟绿萝、鸭脚木的叶片总数 $L$ 、结果较好叶片数 $L^{\prime}$ 以及建模结果的各个评估值. 
表 2 虚拟植物建模结果评估

\begin{tabular}{lccrcc}
\hline \multicolumn{1}{c}{ 植物种类 } & $L$ & $L^{\prime}$ & $P_{L} / \%$ & $m_{P} / \%$ & $m_{L} / \%$ \\
\hline 虚拟绿萝 & 33 & 33 & 100.0 & 99.4 & 98.3 \\
虚拟鸭脚木 & 62 & 48 & 77.4 & 81.6 & 76.5 \\
虚拟花烛 & 10 & 10 & 100.0 & 96.8 & 95.7 \\
\hline
\end{tabular}

对于虚拟植物，通过在多个视角下对植物模 型进行渲染得到对应的图片, 进行叶片识别并剔 除叶片, 最终得到植物完整模型. 图 10 图 12 分别 展示了绿萝、鸭脚木与花烛的建模结果.

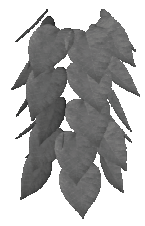

a. 原始图片

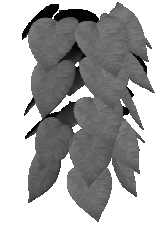

b. 建模结果
图 10 虚拟绿萝的建模结果

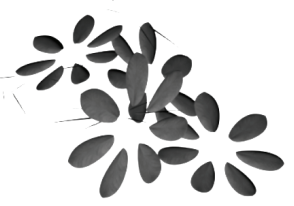

a. 原始图片

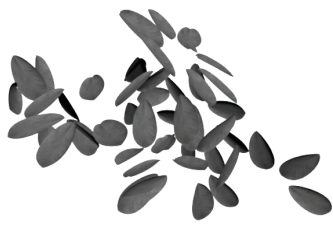

b. 建模结果
图 11 虚拟鸭脚木的建模结果

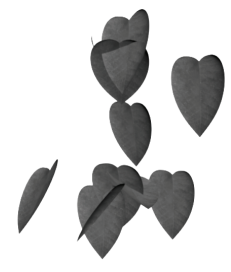

a. 原始图片

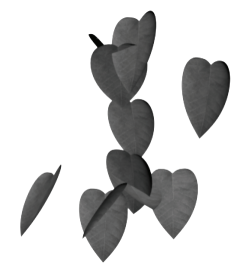

b. 建模结果
图 12 虚拟花烛的建模结果

从表 2 和图 10 图 12 可以看出, 鸭脚木的建 模结果比绿萝与花烛较差, 成功建模叶片占比 $P_{L}$ 、 平均叶片重合度 $m_{L}$ 以及整株植物重合度 $m_{P}$ 上都 较低. 其主要原因是鸭脚木中叶片较多且每片叶 子相较于整株植物过小, 模拟时扫描植物时点云 中的噪声对区分叶片以及模型配准的影响较大. 而绿萝与花烛的叶片尺寸相对整株植物占比大, 噪声对它们的建模结果影响不大.

\section{5 结 语}

针对室内植物的侵人式方法建模过程，本文 提出了一种自动选择被剪掉叶片的方法. 将某一
相机视角下捕捉到的植物 RGB 图片传人叶片检测 网络, 选择出在植物外部且完整可见的叶片. 使用 虚拟叶片数据训练该检测网络, 通过总结真实植 物的叶片分布并使用参数化叶片模型, 模拟出多 株虚拟植物模型并渲染得到大量带标注信息的植 物图片. 基于此, 设计了一种自动化侵人式植物建 模系统, 并在 3 类虚拟植物上进行了测试.

本文方法仍然有可以改进的地方. (1) 对于不 同种类的植物需要总结不同的叶片外形特征与叶 片分布特征, 而且使用的参数化叶片模型只适用 于有单个叶尖类型的叶片. 因此在今后的研究中 可以设计并使用更具有一般化的参数化叶片模型, 将本文方法应用到更多植物中, 使其具有更好的 泛用性. (2) 只使用简单光照模型来渲染叶片生成 训练数据. 已有研究显示, 不同的渲染方法会影响 训练好的模型用于处理真实数据时的性能 ${ }^{[30]}$. 这 方面需要做进一步的探索. (3) 目前以检测到的叶 片的置信度 (即叶片的完整度)为评判标准来选择 带裁剪叶片, 后续可以判断每片叶子所遮挡的信 息量, 选择遮挡信息最多的叶片.

目前本文方法只在虚拟植物上进行了测试, 下一目标是扩展到机器人辅助的真实植物建模上. 虽然整体流程是可行的, 但也面临不少新的挑战. (1) 目前直接将训练好的模型用于真实植物时, 分 割准确率上还不能达到同样精度, 这方面还需要 进一步探索, 以实现高精度地检测真实植物叶片. (2) 需要准确地判断叶片的裁剪点, 并确定机器人 的裁剪操作路径, 使得机器人能自动裁剪叶片; 如 何进行避障以保证机器人在操作时不会触碰到整 株植物是其中的挑战点.

\section{参考文献(References):}

[1] Yin K X, Huang H, Long P X, et al. Full 3D plant reconstruction via intrusive acquisition[J]. Computer Graphics Forum, 2016, 35(1): 272-284

[2] Honda H. Description of the form of trees by the parameters of the tree-like body: Effects of the branching angle and the branch length on the shape of the tree-like body[J]. Journal of Theoretical Biology, 1971, 31(2): 331-338

[3] Prusinkiewicz P, James M, Měch R. Synthetic topiary[C] // Proceedings of the 21st Annual Conference on Computer Graphics and Interactive Techniques. New York: ACM Press, 1994: 351-358

[4] Lintermann B, Deussen O. Interactive modeling of plants[J]. IEEE Computer Graphics and Applications, 1999, 19(1): 56-65

[5] Shlyakhter I, Rozenoer M, Dorsey J, et al. Reconstructing 3D tree models from instrumented photographs[J]. IEEE Computer 
Graphics and Applications, 2001, 21(3): 53-61

[6] Ijiri T, Owada S, Okabe M, et al. Floral diagrams and inflorescences: interactive flower modeling using botanical structural constraints[J]. ACM Transactions on Graphics, 2005, 24(3): $720-726$

[7] Chen X J, Neubert B, Xu Y Q, et al. Sketch-based tree modeling using Markov random field[J]. ACM Transactions on Graphics, 2008, 27(5): Article No.109

[8] Livny Y, Yan F L, Olson M, et al. Automatic reconstruction of tree skeletal structures from point clouds[J]. ACM Transactions on Graphics, 2010, 29(6): Article No.151

[9] Xu H, Gossett N, Chen B Q. Knowledge and heuristic-based modeling of laser-scanned trees[J]. ACM Transactions on Graphics, 2007, 26(4): Article No.19

[10] Bucksch A, Lindenbergh R. C, Menenti M. SkelTre-fast skeletonisation for imperfect point cloud data of botanic trees[C] //Proceedings of the 2nd Eurographics Conference on 3D Object Retrieval. Aire-la-Ville: Eurographics Association Press, 2009: $13-20$

[11] Li Y Y, Fan X C, Mitra N J, et al. Analyzing growing plants from 4D point cloud data[J]. ACM Transactions on Graphics, 2013, 32(6): Article No.157

[12] Yan F L, Sharf A, Lin W Z, et al. Proactive 3D scanning of inaccessible parts[J]. ACM Transactions on Graphics, 2014, 33(4): Article No.157

[13] Xie K, Yan F L, Sharf A, et al. Tree modeling with real tree-parts examples[J]. IEEE Transactions on Visualization and Computer Graphics, 2016, 22(12): 2608-2618

[14] Beardsley P, Chaurasia G. Editable parametric dense foliage from 3D capture[C] //Proceedings of the IEEE International Conference on Computer Vision. Los Alamitos: IEEE Computer Society Press, 2017: 5315-5324

[15] LeCun Y, Bottou L, Bengio Y, et al. Gradient-based learning applied to document recognition[J]. Proceedings of the IEEE, 1998, 86(11): 2278-2324

[16] Girshick R, Donahue J, Darrell T, et al. Rich feature hierarchies for accurate object detection and semantic segmentation[C] // Proceedings of the IEEE Conference on Computer Vision and Pattern Recognition. Los Alamitos: IEEE Computer Society Press, 2014: 580-587

[17] Girshick R. Fast R-CNN[C] //Proceedings of the IEEE International Conference on Computer Vision. Los Alamitos: IEEE Computer Society Press, 2015: 1440-1448

[18] He K M, Gkioxari G, Dollár P, et al. Mask R-CNN[C] // Proceedings of the IEEE International Conference on Computer
Vision. Los Alamitos: IEEE Computer Society Press, 2017: 2980-2988

[19] Cai Z W, Vasconcelos N. Cascade R-CNN: delving into high quality object detection[C] //Proceedings of the IEEE/CVF Conference on Computer Vision and Pattern Recognition. Los Alamitos: IEEE Computer Society Press, 2018: 6154-6162

[20] Chen K, Pang J M, Wang J Q, et al. Hybrid task cascade for instance segmentation[C] //Proceedings of the IEEE/CVF Conference on Computer Vision and Pattern Recognition. Los Alamitos: IEEE Computer Society Press, 2019: 4969-4978

[21] Amara J, Bouaziz B, Algergawy A. A deep learning-based approach for banana leaf diseases classification[M] //Datenbanksysteme für Business, Technologie und WebWorkshopband. Bonn: Gesellschaft für Informatik, 2017: 79-88

[22] Joffe B, Ahlin K, Hu A P, et al. Vision-guided robotic leaf picking[OL]. [2020-05-03]. https://research.qut.edu.au/futurefarming/wp-content/uploads/sites/3/2018/06/Vision-guided-roboticleaf-picking.pdf

[23] Georgakis G, Mousavian A, Berg A C, et al. Synthesizing training data for object detection in indoor scenes[OL]. [2020-05-03]. https://arxiv.org/abs/1702.07836

[24] Ward D, Moghadam P, Hudson N. Deep leaf segmentation using synthetic data[OL]. [2020-05-03]. https://arxiv.org/abs/ 1807.10931v1

[25] Kuznichov D, Zvirin A, Honen Y, et al. Data augmentation for leaf segmentation and counting tasks in rosette plants[C] // Proceedings of the IEEE/CVF Conference on Computer Vision and Pattern Recognition Workshops. Los Alamitos: IEEE Computer Society Press, 2019: 2580-2589

[26] Munisami T, Ramsurn M, Kishnah S, et al. Plant leaf recognition using shape features and colour histogram with K-nearest neighbour classifiers[J]. Procedia Computer Science, 2015, 58: 740-747

[27] Kumar N, Belhumeur P N, Biswas A, et al. Leafsnap: a computer vision system for automatic plant species identification[C] //Proceedings of European Conference on Computer Vision. Heidelberg: Springer, 2012: 502-516

[28] Söderkvist O. Computer vision classification of leaves from swedish trees[D]. Linkoping: Linkoping University, 2001

[29] Chen K, Wang J Q, Pang J M, et al. MMDetection: open MMLab detection toolbox and benchmark[OL]. [2020-05-03]. https://arxiv.org/abs/1906.07155v1

[30] Tsirikoglou A, Eilertsen G, Unger J. A survey of image synthesis methods for visual machine learning[OL]. [2020-05-03]. https://doi.org/10.1111/cgf.14047 